\title{
Comments from young scholars: Can machines completely replace humans in manufacturing processes?
}

C The Author(s) 2018. Published by Higher Education Press. This is an open access article under the CC BY license (http:// creativecommons.org/licenses/by/4.0)

Recently, we asked some young scholars from different fields around the world to send us their comments about this issue:

Can machines completely replace humans in manufacturing processes? If so, how should humans deal with this situation? If not, how can humans achieve a harmonious coexistence with intelligent machines?

Many scholars indicated that machines can only replace humans in repetitive low-skill, complex non-creative and dangerous tasks in manufacturing processes. Humans are needed in high-skilled innovation and critical decisionmaking jobs, as well as in fields that require emotional intelligence. To coexist with machines and achieve good results in the future, humans should make certain changes in education and employee training regime. Humans should also develop principles and standards in dealing with the relationship between humans and intelligent machines. Others held the perspective that humans may be completely replaced by machines in manufacturing processes given that machines have higher capability and better performance to handle most manufacturing processes than humans. Several new types of occupations may emerge in the process of replacement, and the most important thing for humans is to equip themselves with updated and recognized skills and keep pace with the times. We extract their views and suggestions in this study.

Intelligent automation is increasingly being used in manufacturing processes around the globe. These machines perform many tasks that were once performed by humans. A study from Oxford Martin School (2016) used data from OECD and reported that $57 \%$ of jobs on

Received August 3, 2018

Shanlin YANG ( $(\bowtie)$

School of Management, Hefei University of Technology, Hefei 230009, China

E-mail: yangs1@hfut.edu.cn average are susceptible to automation and this value reached $77 \%$ in China. In a press release by Guangdong Everwin Precision Technology, one of the company's representatives commented that 'by using 60 industrial robots, an assembly line that needed about 600 workers in the past now needs only 100 .'

Automation development in manufacturing processes increased the number of new types of jobs. Most futurists predict that future jobs in manufacturing can shift from low-skilled, repetitive jobs to programming and coding jobs for industrial automation. Moreover, recent technological advances favored certain skill groups over others. Employees are required to adjust to the rapid changes in manufacturing systems and continuously update their knowledge.

The adoption of 'co-bots', which are collaborative robots that can work alongside human workers in a plant, is also increasing. A recent report by the International Federation of Robotics indicated that future human-robot collaboration will help obtain a flexible production of complex products in small quantities.

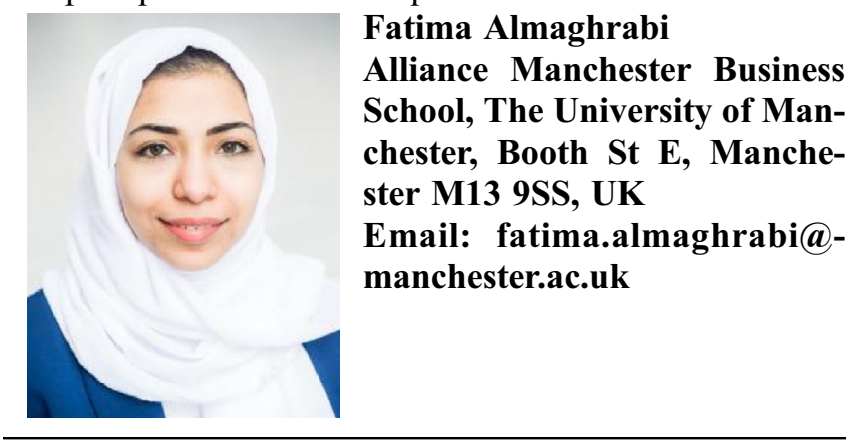

The 1980s witnessed the birth of a new type of medicine described as biopharmaceuticals, which are capable of treating severe conditions such as hemophilia, arthritis and certain cancers. However, the molecules involved in these medicines are proteins, which are significantly larger and more complex than usual drugs such as aspirin. Thus, 
sophisticated manufacturing processes are required. The complexity of these processes steams from the fact that living cells must be genetically modified, which results in fragile architectures that require stringent storage, preprocessing, and purification regimes.

At present, the biopharma industry is the fastest growing industry with revenues that exceed $\$ 150$ billion globally. The industry is currently facing the same challenges as other businesses. These challenges include competitiveness in terms of cost, quality, and market speed. Companies handle these challenges by using novel manufacturing process technologies, such as continuous and single-use technologies. Advances in robotics and real-time process monitoring combined with sophisticated control algorithms and digital simulation models are slowly finding their way into the industry, which contribute to increasing product quality and efficiency.

Despite these developments, the complete replacement of humans in the manufacturing of biopharmaceuticals remains unimaginable. The idea of outsourcing to machines the production of medicine that can decide over death or life is simply too risky for patients. However, the push in digitalization can radically change the industry in a way that requires employees to shift their focus from undertaking repetitive manual tasks to using analytics and computer modeling. Thus, control and throughput improve through real-time monitoring of process performance. The key to achieving this transformation is to convince employees of new technologies and provide them with a well-thought training regime to obtain the required digital skills. Safoni's digital biologics plant shows that this transformation is possible and I am convinced that many similar plants may follow.

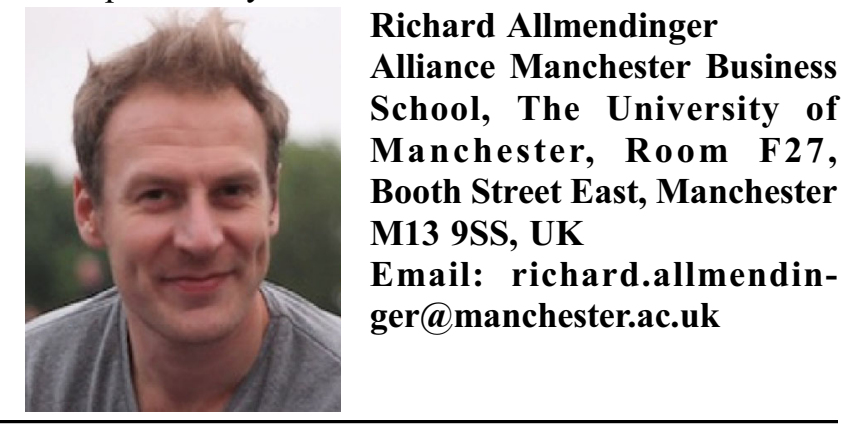

The global manufacturing industry is moving toward its next automation breakthrough by incorporating artificial intelligence (AI). The next generation of robotic machines can supersede human involvement in routine physical and cognitive skills. These machines can also be trained to perform complex tasks and provide trustworthy pieces of advice. These machines can be less dependent on humans than before. They can improve by learning new things and patterns from initial inputs from humans and from data gathered on their own. However, these factors cannot make humans obsolete. Instead, humans may focus on highskilled jobs that involve innovation and strategy. The transition to automated labor market can create areas of employment. Smartphone innovation and increased processing power of computers created many jobs related to data science, application development, information security and social media.

Overall, automation may cause redundancy of lowskilled jobs. However, automation can drive workforce toward supervision, repair and maintenance of new systems, as well as error and data interpretation jobs. Manufacturing firms face the challenge of training their workers for their future engagement with new machines. They should initiate the process to identify skills that future machines may not be able to replicate in their factories. The identified skill gaps in workers can be consummated by providing generalized education to perform tasks in their automated factories. Schools and universities should be prepared to provide knowledge and skills to work in the automated world. They can focus on traits that machines cannot truly outperform humans, such as critical thinking, creativity and emotional intelligence. In the future, humans and robotic machines must learn to collaborate to achieve superior results compared with what either automated machines or humans can accomplish alone.

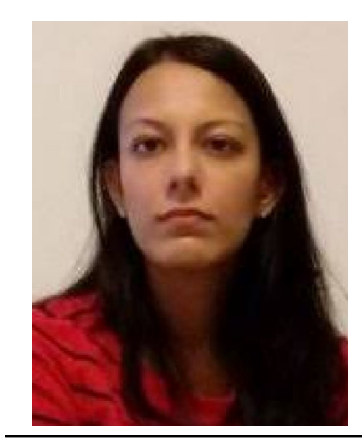

Swati Sachan

Alliance Manchester Business School, The University of Manchester, F39, 2 Booth Street, Manchester, M13 9SS, UK

Email: swati.sachan@manchester.ac.uk

Philip K. Dick introduced the concept of AI production in his novel "Autofac" in 1995. Human beings have been continuously realizing this great dream with the progress of science and technology. In the past 20 years, human auto manufacturing capacity has increased rapidly. Various industrial robots and $\mathrm{CNC}$ machines have continuously worked together as producers. FANUC realized the light out factory in 2001; this kind of pure machine-manufactured factory is constantly being promoted. The advantages of this technology are absence of fatigue, no need for rest, lack of labor disputes, few operational errors, precise machining accuracy, fast production speed, and low labor costs. These advantages transformed unmanned factories into an inevitable trend. Many people are anxious toward this development. They believe that unmanned plants can result in unemployment, which in turn can lead to further social problems, such as a huge gap between the rich and the poor and serious confrontation among social classes.

However, excessive worrying is unnecessary. Humans underwent a long civilization. They have experienced several technological revolutions in the past thousands of 
years. Each technological revolution means transfer of labor from one industry to another. For example, the industrial revolution unleashed heavy labor from small workshops and created additional services, finance, and education. Humans cannot be completely replaced by machines in this wave of AI. Similar to previous technological revolutions, machines further help human complete repetitive and cumbersome work. Human labor can be transferred to industries that require high creativity, such as cultural and technology R\&D industries. Machine intelligence does not have a negative impact on human society. This intelligence promotes the speed of human science, technology, and cultural development because labor is released from low-level large-scale manufacturing to creative industries.

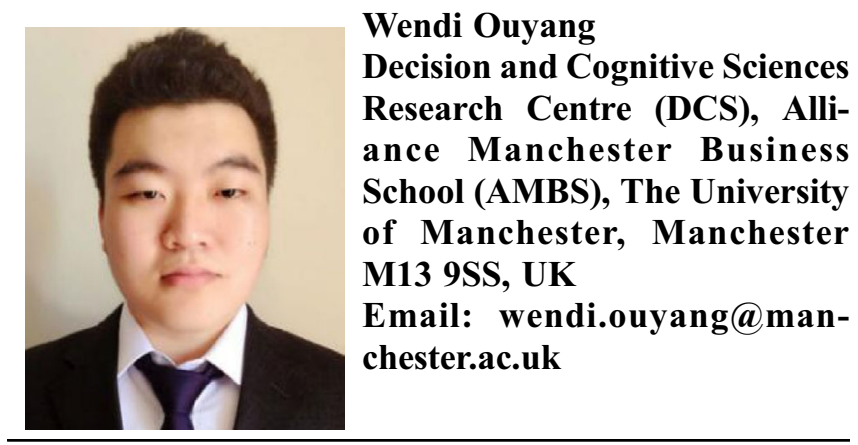

The boom of AI technology boosts the evolution of intelligent machines, which can emancipate the productive forces of humans. Thus, people worry that all workers will one day be replaced by intelligent machines in factories. However, I do not think this situation will occur because realizing complete automation is still full of technological challenges. Moreover, manufacturing processes and humans are two inseparable parts. Putting these two reasons together can certainly lead to the long-term coexistence of humans and machines in manufacturing processes. My concerns toward achieving a harmonious coexistence environment for them are threefold.

A fundamental principle should always be established and followed (i.e., Humans Dominate Machines). We must advocate the absolute leading position of humans; otherwise, humans are exposed to unbearable threats or unpredictable dangers. Disastrous effects may arise, such as depression, recession, or disturbance.

Division of labor is another critical factor that should be considered. Previously, we use machines to perform repetitive, tedious and dangerous tasks. I think this original intention should be held for a long time in the future. A labor framework in which are humans located at the top level and machines are located at the bottom level may be necessary. Building this labor division framework also comes with a basic principle.

Intelligence management, which I think is important, is not used in our methodologies. Machine intelligence can develop uninterrupted as technology develops. We should pay enough attention to this problem, whether machines exceed us on intelligence or not. We may need an intelligence standard, evaluation grade of practical intelligence and a third-party supervisory organization for intelligent machines which we prepare to launch into our factories. 'Machine Build Machine' can be acceptable, whereas 'Machine Build Intelligence' should be strictly forbidden.

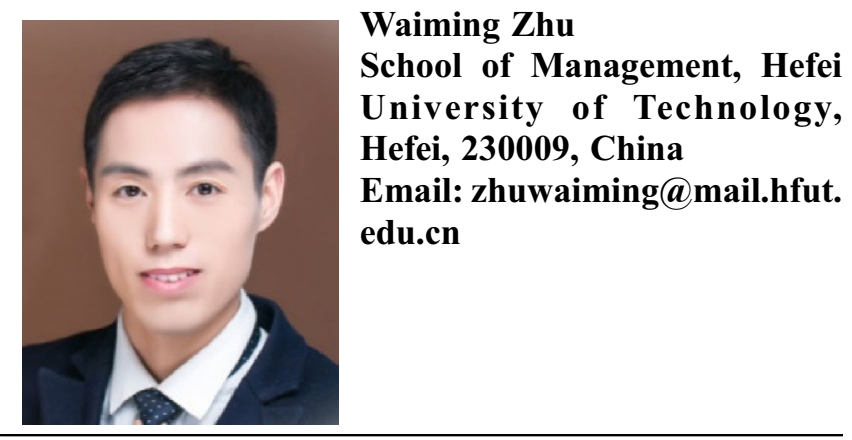

Every century has its own technological revolution. The most recent ones, the Industrial Revolution in the 19th century and the Digital Revolution in the 20th century, are followed by the fear of the unknown that can inspire attacks against machines. Therefore, the discussion on technology replacing humans has been pursued for two centuries. This debate is centered on the following question: What is the upcoming technological revolution of the 21st century?

Robotics not only reduces manufacturing cost, but also prevents the most common accidents at work, such as slips, trips, falls, muscle strains and being hit by falling objects. In 2016 and 2017, over 600000 non-fatal injuries were reported at work in the UK. Missed days at work due to work injuries is a major economic issue. Low-skilled human labor is being steadily replaced by the help of technology and creates several high-skilled work places. Computerisation is an example.

The major change in the understanding of jobs in the 21 st century is that workers do not want to perform repetitive, dull jobs that do not engage with their personalities. Management in enterprises has a new focus in terms of employee development enhancing creativity, specialized skills and communication. I believe that the arrival of high-end technology pushes humanity to acquire further knowledge and seek 'out of the box' solutions for problems. In addition, machines can quickly generate large data and find information in seconds. Machines do not reach the level of humans in analyzing and interpreting such data. Therefore, technology-based higher education should be accessible and attractive for people of any age to pursue and fulfil the demand for skilled employees.

In summary, machines should not be envisioned as an enemy but as a tool for a productive, cost-effective and safe work. 


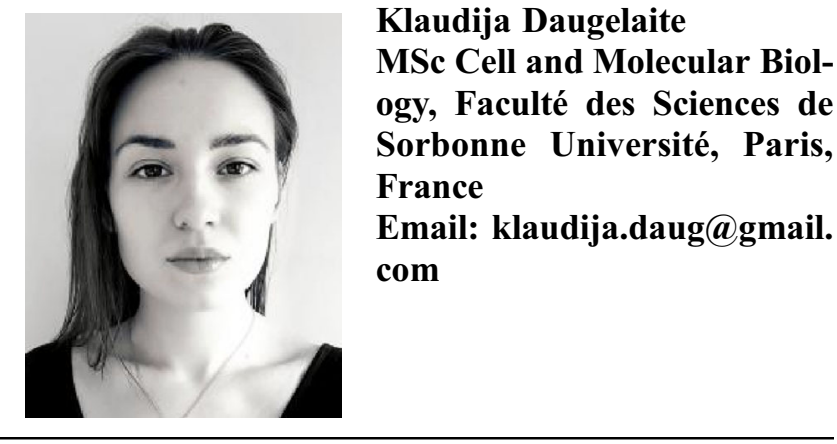

Intelligent machines attain faster production rates than people, and the products produced by machines have higher quality and precision than that by humans. Thus, I believe that machines can completely replace humans in manufacturing processes in the future. The good side is that intelligent machines can make up for the labor shortage caused by the aging population or increasing cost of human resource. The bad side is that the rapid development of intelligent machines can result in unemployment of manufacturing workers. However, we should not extremely worry about this matter. This phenomenon can create new jobs. For example, the job of maintaining intelligent machines is a gradual process, wherein intelligent machines completely replace people. Therefore, we have enough time to cope with the bad effects of this phenomenon. Manufacturing enterprises must provide good training for employees to enable the latter to develop competence for advanced design and maintenance or creative work in the future. Moreover, manufacturing enterprises should do well in talent and technology reserve to cope with the impact of $\mathrm{AI}$ on manufacturing.

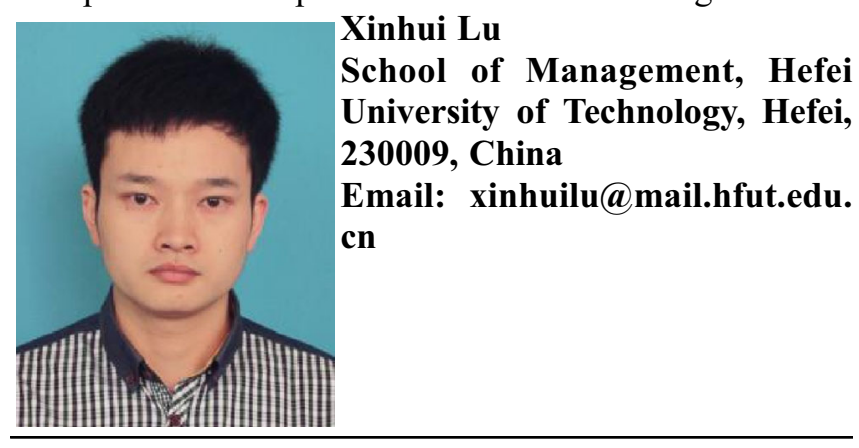

"One machine can do the work of fifty ordinary men, but no machine can do the work of one extraordinary man." Elbert Hubbard wrote this passage. However, machines have become smart after 100 years. They can interact with humans, analyze data, and make suggestions in a fraction of a second. In manufacturing processes, machines have replaced humans to a huge extent, and machines are doing the job more efficiently than humans. Thus, many people believe that machines can completely replace humans.

Machines, regardless of their capability and prowess, can only do what they are programmed to do. Nowadays, machines can solve difficult problems and perform arduous tasks better than humans. However, the objectives are always set by humans. Machines then try to achieve predefined goals or maximize objective measures. Intelligent machines can become increasingly good at finding optimal ways to reach objectives in the mold created by humans.

Therefore, machines, which have largely replaced humans, demand that users must have great expertise and intellectual capacity. The forthcoming prevalence of intelligent machines necessitates future generations to be properly trained on how to interact with machines. Operating machines will evidently not be a simple task as it will require a set of skills that will likely be the subject of specialized training sessions, if not programs.

Intelligent machines can perform tasks for which human presence was considered indispensable until recently. As machines become increasingly intelligent and replace humans in increasing rates, humans may have to tailor their skills for the new reality.

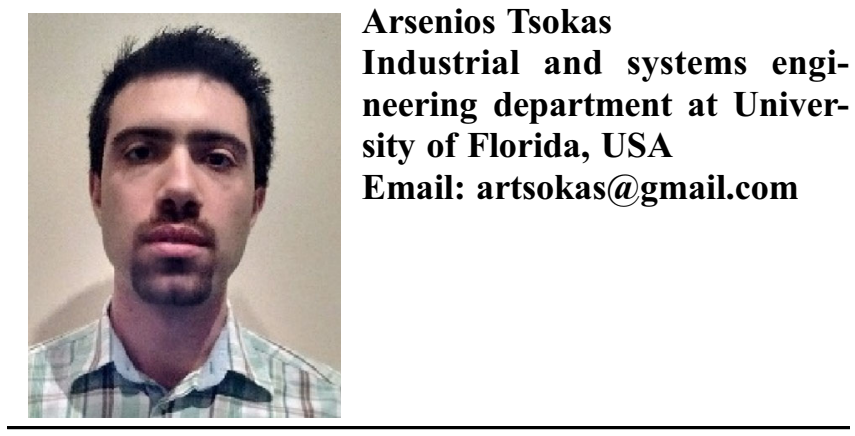

Manufacturing technologies have improved and demands for new technologies have increased. Thus, automation or use machines instead of human became an important issue. Some people believe that manufacturing systems cannot reach the point wherein everything is completely done by machines. Others subscribe to the view that machines have certain abilities that make them work better than before and can be considered a good replacement for human workers. I vote for the latter choice.

One of the compelling reasons is the higher capability and better performance of machines to handle most manufacturing processes than humans. However, what I previously mentioned may not be overgeneralized to all contexts. In addition, products in certain industries related to information technology require high precision and accuracy, which humans cannot reach most of the time in mass production. Let me provide another example to further elaborate the idea. Let us consider industries where workers must work with dangerous or radioactive chemicals. Robots can be a good alternative to protect human workers and keep them safe. The cost of broken robots is nothing compared with a small injury in humans. Manufacturers should consider the highest priority for human safety above anything else.

Moreover, new issues can emerge through automation, 
such as dealing with unemployed workers. This shift from humans to machines should not suddenly take place. Otherwise, many workers with no expertise end up dealing with new situations. They are required to be trained to supervise processes and check outputs that machines cannot do at certain levels.

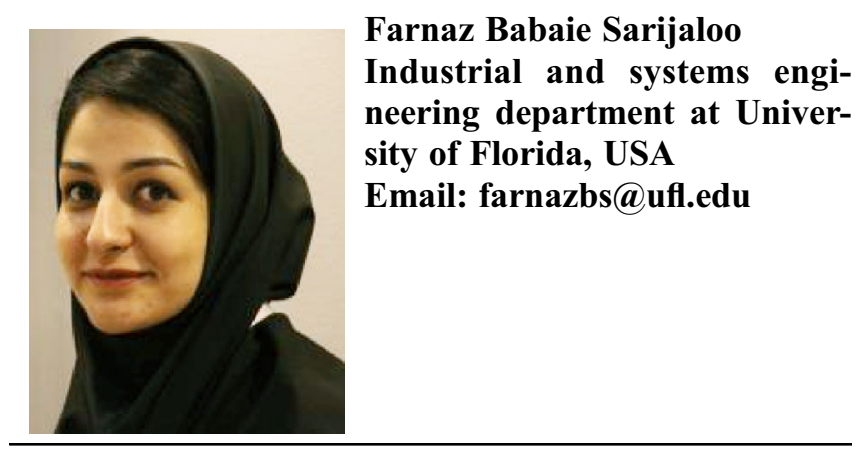

Low-technology industries are currently being automated. Part of human labor in high-technology manufacturing industries has also been replaced by machines. However, machines may never completely replace humans in manufacturing processes in the long run because the complexity of the products people need and rely on increases as technology advances. This scenario is possible if AI does not reach super intelligence level. Thus, machine intelligence cannot obtain greater than the intelligence of all the human race combined. In this scenario, we may have serious problems to handle.

If $\mathrm{AI}$ is confined due to incapabilities or human restrictions, part of the manufacturing industry must deal with the manufacturing of products in cutting-edge technologies. The complexity of these products may require at least human supervision as humans are the only ones who can deal with problems of scientific complexity. Therefore, methods and the processing in manufacturing industries may become automated in the future, but the cutting-edge equipment that humans require for their future endeavors and aspirations, such as hyperloops, space exploration and illness treatments may require human intelligence.

Humans may need to deal with those advancements by spending more of their lives learning and less on working. Thus, school and university years should increase so that people can acquire enough knowledge before joining the workforce. Moreover, people do not have to deal with unemployment issues because they will start working later in their lives, thereby reducing the supply of workforce. However, material goods that people in the workforce produce may be more than enough for the working people, as well as those in the educational phase.

If AI does not become super intelligent, then hightechnology manufacturing industries may always need humans to solve problems. Human imagination may also be necessary to make updates on current equipment. A harmonious coexistence of humans and machines can be a result of humans being adequately educated on hard sciences and creativity so that they can solve any problem that may arise.

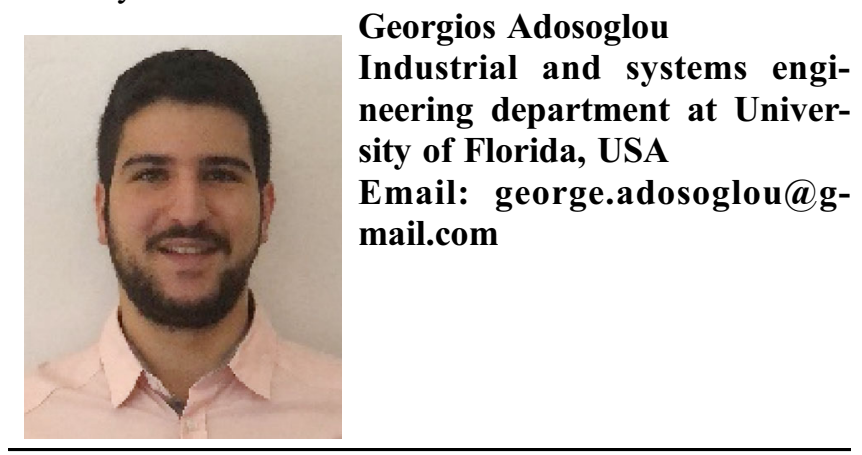

Machines in manufacturing processes have been developed in two ways. The first approach is to control all variables through scheduling. The other is to adapt and respond to changes in a certain situation and environment. The former takes up most of the philosophy of the existing industrial robots. Nowadays, developments of AI in accordance with utilization of big data through sensors are progressing gradually in the context of Industrial Revolution 4.0.

Is it possible to be completely replaced by machines through the development of AI and the use of big data? The AI developed so far is referred to as 'weak AI', which means a weak inference in a narrow task. Accumulating all data in different natural disaster situations is impossible in practice. How can we deal with an earthquake that occurs once in 50 years? How can we cope with the largest storm in 100 years? Only small data can be accumulated. We cannot expect machines to adapt to such unfamiliar . situations. Thus, I think that machines cannot replace humans perfectly in the future.

However, I think that the type of work human does can change rapidly. The human labor force, which has been used in the process of production directly, may end up supporting machines. Humans may also train machines' AI and supervise it. In conclusion, the role of human as a computer scientist can be expanded to assist machines in manufacturing processes.

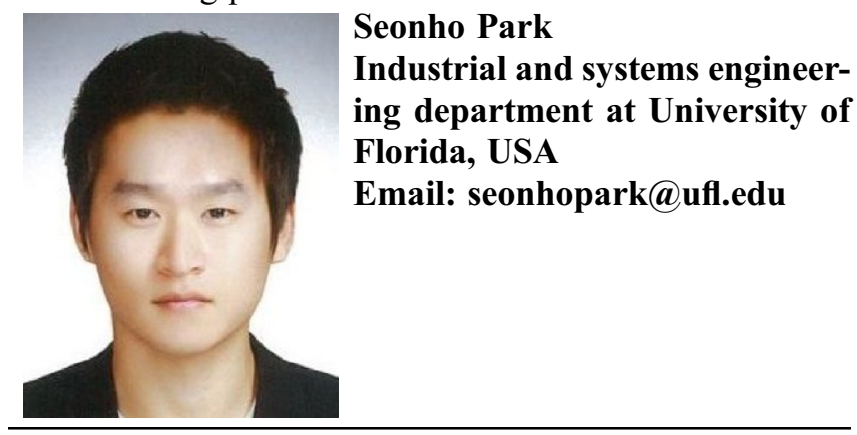

In my view, machines with full intelligence can completely replace human labor in production lines, but the creativity and spiritual power of humans cannot be completely replaced in the whole manufacturing process. A new upheaval in manufacturing industry is being led by 
a new wave of intelligent and automation boom. The core of such an upheaval includes artificial identification technology, Internet of things, machine learning and distributed intelligence. Machines can touch all kinds of normalized work with the development of these technologies from manual work to mental work. Machines, such as automatic textile and punching machines, can do better than humans. In addition, machines, which humans have trained, can do what humans cannot do, such as highprecision computer chip-making machines and molten glass-blowing machines. However, to pursue original innovation and spiritual enjoyment in manufacturing processes, humans cannot and may not be completely replaced by machines. Humans create machines to liberate themselves from tedious work, but machines never know where humans want to go for the next step. Moreover, China advocates a spirit of craftsmanship, which means dedicated, lean, focused, and innovative. Artisans like to carve their products, improve their craft, and enjoy the process of product sublimation in their hands. They are not completely dependent on machines in manufacturing processes due to emotional needs.

Never view automation and intelligent trends as a competition with machines. This trend should be viewed as humans and machines going forward hand in hand. Machines may take over your job, but humans can create new jobs for you, such as an intelligent machine maintenance engineer. Intelligent machines may be partners who are essential to the completion of your jobs and may help you get paid more than before.

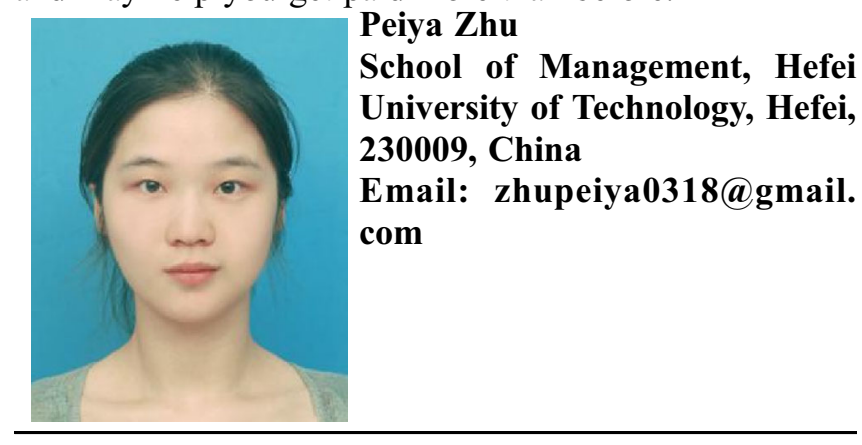

I believe machines can completely replace humans in the future. Such a replacement has already occurred in the past decades. In the last century, many professions, such as typists, blacksmiths, and telephone operators, disappeared. According to a survey conducted by the University of Cambridge, approximately $47 \%$ of the 702 jobs in the US will be gone in the next 10 to 20 years. The pace of future career changes can increasingly become fast given the rapid development of science and technology. However, we do not have to panic about it. Technological advances, while spurring the demise of traditional work, have spawned many new jobs, such as network engineer, data algorithmic engineer, data analyst, data mining engineer, data product manager, data architecture engineer, big data visualization engineer, big data crawler engineer, and other jobs that are dependent on Internet and big data. Most of these jobs were unfathomable in the early 20th century. Now, we should ensure that we will not be eliminated by technology in the future. Thus, we must learn as much as we can to increase opportunities to obtain high-quality jobs.

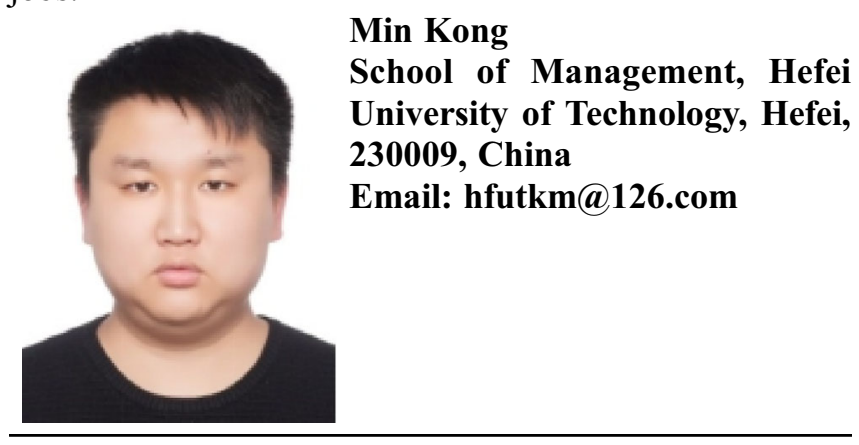

I think that intelligent machines can, has been replacing, and will replace humans completely in manufacturing processes if 'humans' here represent laborers who perform physical activities or operate machinery in a predictable environment. Under both situations, machines can and will replace humans because machines are cheap, efficient, bring good quality and make few errors. However, manufacturing processes may involve unpredictable physical and intelligent work, such as repairing and diagnosing, in which humans are needed.

Many people usually panic when wide automation and robotics are approaching. Why? We are worried that if machines and robots replace human labor, then many people may lose jobs. Certain jobs are disappearing. This occurrence does not mean that those freed people should starve in streets because they were replaced by machines. Certain jobs may disappear, but other new jobs may appear. People who occupations are disappearing can join possible new sectors created for automation and robotics. For example, robots can be aged and certain sectors may recycle them. Thus, when intelligent machines greatly affect manufacturing processes, the challenge we must face is not machines replacing humans but self-learning and self-changing to be well prepared. In addition, we should think about how much pain automation and robotics have helped relieve as they free people from huge and repetitive work.

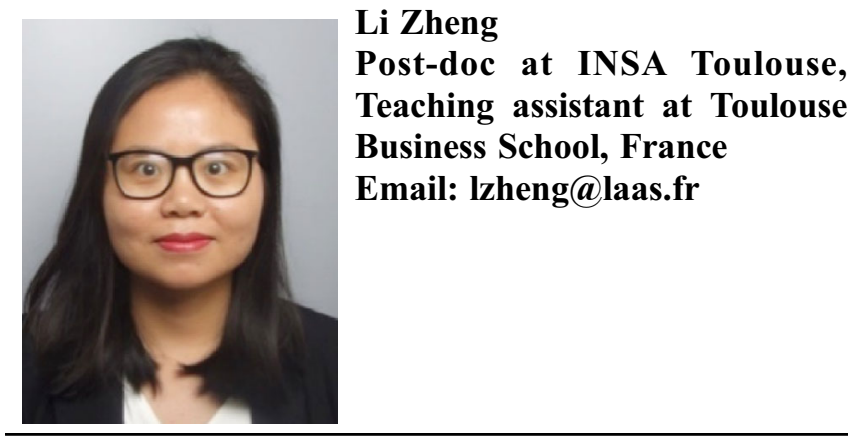

Machines can never be fully autonomous from humans 
who initially designed them. Factory machines are not smart yet, but they are programmed by humans to reach two objectives. The first objective is to control and assist workers, and the second one is to replace workers. The first case does not involve substitution of employees. Thus, no major human/machine coexistence issues are raised. Human maintenance is always present in the integration of machines in factories. For the most modern factory, this team can work remotely in a hub, wherein all the knowhow concentrate on controlling many factories. Machines replacing workers' job is a global trend as mechanization is used to reduce agriculture labor. These job market changes will primarily affect workers whose job is threatened. The increase in companies' productivity can imply a decrease of workers' working time. Workers are also trained to be maintenance technicians. Finally, when machines help workers, the former must have a user-friendly intuitive user interface. Recent developments of augmented reality, voice command, and virtual assistants can be applied to omit the pain points of factory workers.

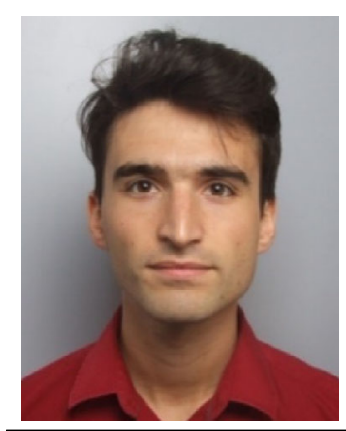

Maxime Ruffel

Calibration Engineer at FEV France for Renault, France Email: ruffel@alumni.insatoulouse.fr 